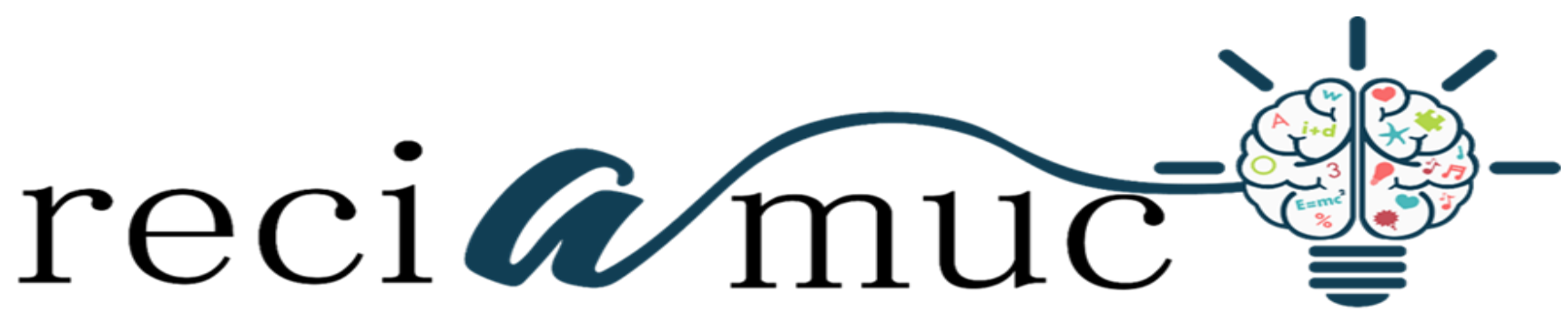

Revista científica de investigación actualización del mundo de las ciencias

Gabriela Cecilia Arroyo Calvopiña a ; Anthony James Chávez Salazar ${ }^{\text {b; }}$ Jessica Fernanda Toledo Cascante ${ }^{c}$; Silvia Jimena Zambrano Salvatierra ${ }^{d}$; Fabricio Wladimir Gutiérrez Rivera ${ }^{e}$; Michelle Madeleyne Salazar Bustamante ${ }^{f}$

Complicaciones postoperatorias a las pacientes sometidas a una mastectomía

Postoperative complications to patients undergoing a mastectomy

Revista Científica de Investigación actualización del mundo de las Ciencias. Vol. 3 núm., 4, octubre: 2588-0748, 2019, pp. 203-226

DOI: 10.26820/reciamuc/3.(4).octubre.2019.203-226

URL: http://reciamuc.com/index.php/RECIAMUC/article/view/407

Código UNESCO: 3205 Medicina Interna

Tipo de Investigación: Artículo de Revisión

(c) RECIAMUC; Editorial Saberes del Conocimiento, 2019

Recibido: 15/05/2019

Aceptado: 07/08/2019

Publicado: 01/10/2019

Correspondencia: gaby_arca@hotmail.com

a. Médica Cirujana; Médico general en funciones hospitalarias en el servicio de neonatología - Hospital general Marco Vinicio Iza; Lago Agrio - Ecuador; gaby arca@hotmail.com

b. Doctor en Medicina; Médico Residente de Anestesia Hospital Marco Vinicio Iza; Lago Agrio - Ecuador; anthonyjames.med@gmail.com

c. Médico General; Médico Residente en el área de emergencia - Hospital general Marco Vinicio Iza; Lago Agrio - Ecuador; fertoledoc1@gmail.com

d. Médica Cirujana; Médico residente del Hospital Básico San Andrés; Flavio Alfaro - Ecuador; silviajimena1993@ gmail.com

e. Médico Cirujano; Médico cirujano en función hospitalaria - Hospital Básico Jipijapa; Jipijapa - Ecuador; wladiguti@hotmail.com

f. Médica General; Hospital Gustavo Domínguez; Santo Domingo - Ecuador; michellesalazarbustamante@ gmail.com 


\section{Complicaciones postoperatorias a las pacientes sometidas a una mastectomía}

Vol. 3, núm. 4., (2019)

Gabriela Cecilia Arroyo Calvopiña; Anthony James Chávez Salazar; Jessica Fernanda Toledo Cascante; Silvia Jimena Zambrano Salvatierra; Fabricio Wladimir Gutiérrez Rivera; Michelle Madeleyne Salazar Bustamante

\section{RESUMEN}

El cáncer de mama es una enfermedad crónica degenerativa, lo que conduce a buscar actualmente nuevos conocimientos sobre las causas que lo originan, con el fin de luchar con esta enfermedad, en consecuencia, se ha determinado que la piedra angular más importante para reducir esta problemática es la prevención y detección temprana, pues, al detectar precozmente, se establece un diagnóstico adecuado y un tratamiento con altas probabilidades de curación; pero sí se hace tardíamente es poco probable que se pueda ofrecer un tratamiento curativo. Es decir, las mujeres diagnosticadas en estadios avanzados, para mejorar su salud y calidad de vida, se fija como tratamiento de elección el quirúrgico, como lumpectomía, cuadrantectomía, mastectomía simple, mastectomía radical modificada y mastectomía radical o total, además, frecuentemente, se hace disección ganglionar para prevenir y determinar sí coexisten ganglios positivos a metástasis, con el fin de asignar tratamientos adyuvantes para erradicar la enfermedad. Por lo tanto, su naturaleza, requiere que la mujer reciba tratamiento multimodal como la cirugía para su control, exponiéndola a numerosos riesgos que la llevan a presentar complicaciones postoperatorias entre las cuales se pueden mencionar: infección de herida quirúrgica que puede provocar deshiscencia, también se encuentra el seroma, ocasionada en su mayoría por el manejo ineficaz de drenajes; dolor; hematoma, relacionado por el uso de anticoagulantes; y el linfedema, asociado a la disección ganglionar, además de la suma de algunas comorbilidades como obesidad, hipertensión y falta de adherencia a conductas de disminución de riesgo. Aspectos, de importancia para el desarrollo del artículo, mediante el cual se llega a analizar las complicaciones postoperatorias a las pacientes sometidas a una mastectomía, para ubicarlo en una investigación documental y elaborar las conclusiones pertinentes.

Palabras Claves: Complicaciones Postoperatorias; Mastectomía; Enfermedad Crónica Degenerativa. 


\title{
Complicaciones postoperatorias a las pacientes sometidas a una mastectomía
}

Vol. 3, núm. 4., (2019)

Gabriela Cecilia Arroyo Calvopiña; Anthony James Chávez Salazar; Jessica Fernanda Toledo Cascante; Silvia Jimena Zambrano Salvatierra; Fabricio Wladimir Gutiérrez Rivera; Michelle

Madeleyne Salazar Bustamante

\begin{abstract}
Breast cancer is a chronic degenerative disease, which leads to today's search for new knowledge about the root causes, in order to fight with this disease, consequently, it has been determined to be the most important cornerstone for reducing this problem is prevention and early detection, as early detection establishes an adequate diagnosis and treatment with a high probability of cure; but it is done belatedly it is unlikely that curative treatment can be offered. That is, women diagnosed at advanced stages, to improve their health and quality of life, are set as a treatment of choice surgical, such as lumpectomy, quadrrectonectomy, simple mastectomy, modified radical mastectomy and radical mastectomy or in addition, gangliond dissection is often done to prevent and determine whether positive nodes co-exist for metastasis, in order to assign adjuvant treatments to eradicate the disease. Therefore, its nature requires that the woman receive multimodal treatment such as surgery for her control, exposing her to numerous risks that lead to postoperative complications among which can be mentioned: wound infection that can cause dehiscence, there is also seroma, mostly caused by ineffective drainage management; pain; hematoma, related to the use of anticoagulants; and lymphedema, associated with ganglion dissection, in addition to the sum of some business-like styvencies such as obesity, hypertension and lack of adherence to risk-decreasing behaviors. Aspects, of importance for the development of the article, through which postoperative complications are analyzed to patients undergoing a mastectomy, to locate it in a documentary research and draw relevant conclusions.
\end{abstract}

Key Words: Postoperative Complications; Mastectomy; Degenerative Chronic Disease. 


\section{Complicaciones postoperatorias a las pacientes sometidas a una mastectomía}

Vol. 3, núm. 4., (2019)

Gabriela Cecilia Arroyo Calvopiña; Anthony James Chávez Salazar; Jessica Fernanda Toledo Cascante; Silvia Jimena Zambrano Salvatierra; Fabricio Wladimir Gutiérrez Rivera; Michelle Madeleyne Salazar Bustamante

\section{Introducción.}

La mama en reposo está constituida por 6-10 sistemas principales de conductos, cada uno de los cuales está dividido en lobulillos, unidades funcionales del parénquima mamario. Cada sistema ductal drena a través de una vía excretora independiente o seno lactífero. Las sucesivas ramificaciones de los conductos galactóforos en dirección distal terminan en los conductos terminales. La areola, pezón y desembocaduras de los conductos galactóforos principales están revestidos de epitelio escamoso estratificado. El revestimiento de los conductos mamarios principales se convierte en un epitelio columnar pseudoestratificado y después en un epitelio cuboidal de dos capas.

Por debajo del epitelio de revestimiento, más prominentemente, puede verse una capa baja de células aplanadas: las células mioepiteliales. Dichas células contienen miofilamentos orientados paralelamente al eje largo del conducto. Siguiendo escrupulosamente el contorno de los conductos y conductillos existe una membrana basal. En relación al planteamiento, Torrealba y Minota (2017), destacan que el riesgo de padecer un cáncer de mama aumenta con la edad, siendo el riesgo estimado de padecerlo a lo largo de la vida para una mujer blanca de 13,1\%; desde el momento del nacimiento, la probabilidad de fallecer de un cáncer de mama es de 3,4\%. Asimismo, complementan que es el más frecuente en la mujer, representando el $31 \%$ de todos los tumores de la población femenina. Se estima que una de cada ocho mujeres que alcancen la edad de 85 años habrá desarrollado cáncer de mama en el curso de su vida.

Es importante acotar, que la incidencia de la enfermedad tiene variaciones geográficas significativas, es más frecuente en países del Norte de Europa, Estados Unidos y Canadá siendo muy baja en países orientales cómo China, Japón y Corea, estas variaciones parecerían deberse más a factores ambientales, de costumbres y hábitos alimentarios que a factores raciales. Se ha visto que los grupos de orientales que emigran a Estados Unidos, luego de la segunda generación, presentan una incidencia similar a la del país huésped. En los últimos años se ha originado un incremento en las tasas de cáncer de mama en los países orientales, probablemente por las modificaciones de sus hábitos de vida con mayor semejanza a occidente. 


\section{Complicaciones postoperatorias a las pacientes sometidas a una mastectomía}

Vol. 3, núm. 4., (2019)

Gabriela Cecilia Arroyo Calvopiña; Anthony James Chávez Salazar; Jessica Fernanda Toledo Cascante; Silvia Jimena Zambrano Salvatierra; Fabricio Wladimir Gutiérrez Rivera; Michelle

Madeleyne Salazar Bustamante

Las nuevas investigaciones registradas a nivel mundial, muestran que las estadísticas sobre incidencia, prevalencia y mortalidad por cáncer en el mundo mostraban 10.900 .000 casos nuevos por año con 6.700 .000 muertes, con 24.700 .000 personas viviendo con cáncer dentro de los 3 años del diagnóstico. Los más frecuentes son pulmón con 1.350 .000 casos y mama 1.150 .000 casos anuales. El de mama es el más prevalerte en el mundo con 4.500 .000 mujeres sobrevivientes dentro de los 5 años del diagnóstico. En América Latina, las tasas de mortalidad por cáncer mamario están en aumento y registran niveles sin precedentes en muchos países, como Colombia, Costa Rica, México, Puerto Rico, Uruguay y Venezuela.

En el caso específico del cáncer de mama existen muchos factores de riesgo por lo que resulta muy difícil establecer un responsable único, es por ello que se habla de un origen multifactorial, familiar, hormonal, ambiental, entre otros. Estos factores son características que con mayor o menor frecuencia presentan las pacientes portadoras de la enfermedad. Si bien los mismos pueden influir en el desarrollo del cáncer, la mayoría no es una causa directa de esta enfermedad. Siendo los factores de riesgos aquellos que pueden provocar efectos adversos a la salud, y depende del tiempo de exposición al mismo o la predisposición genética de cada individuo.

Algunas personas que tienen varios factores de riesgo nunca desarrollan cáncer, mientras que sí lo hacen otras personas sin factores de riesgo conocidos. Muchos casos de cáncer de mama se presentan en mujeres sin factores de riesgo evidentes y sin antecedentes familiares de cáncer de mama. Esto significa que todas las mujeres deben estar atentas a los posibles cambios en las mamas realizándose el autoexamen con regularidad y hablar con su médico para realizarse exámenes clínicos y radiológicos como la mamografía, con la finalidad de hacer un diagnóstico precoz de alguna enfermedad o plantearse la posibilidad que más de un factor de riesgo influya en el desarrollo del cáncer.

En cuanto al tratamiento del cáncer de mama se sigue un protocolo, normas y pautas, establecidas en base a la experiencia científica que se tiene en el tratamiento de este tumor. Estos protocolos, recogen las indicaciones o limitaciones del tratamiento en función de los siguientes factores: edad del paciente, estado general y hormonal (premenopausia, menopausia), localización del tumor, 


\section{Complicaciones postoperatorias a las pacientes sometidas a una mastectomía}

Vol. 3, núm. 4., (2019)

Gabriela Cecilia Arroyo Calvopiña; Anthony James Chávez Salazar; Jessica Fernanda Toledo Cascante; Silvia Jimena Zambrano Salvatierra; Fabricio Wladimir Gutiérrez Rivera; Michelle Madeleyne Salazar Bustamante

fase o estadio en la que se encuentra la enfermedad, receptores hormonales del tumor, grado de las células y positividad para algunos factores biológicos. El tratamiento propuesto por el especialista no va a ser el mismo en todos los pacientes. Los más frecuentemente empleados en el cáncer de mama son la cirugía, radioterapia, quimioterapia y hormonoterapia.

Por ello, al realizar la mastectomía como proceso quirúrgico provoca alteraciones en determinados aspectos concretos de la imagen corporal, como sentimientos negativos en torno a la propia desnudez y devaluación del sentimiento de atractivo físico. Una de las consecuencias más notorias de la mastectomía es el Impacto psicológico por no saber en qué va a desencadenar el diagnóstico del cáncer, mastectomía y reconstrucción, situación que se manifiesta en la paciente con el stress e incertidumbre; pues, la pérdida de una o ambas mamas trae para la mujer sufrimientos psicológicos. Estos dependerán de su edad, hijos, esposo, trabajo y grado de importancia que ella les atribuya a las mamas antes de la mastectomía, y se pueden manifestar con una serie de síntomas como ansiedad, insomnio, vergüenza, sentimientos de inutilidad, minusvalía, y para la mayoría de las mujeres representa una crisis personal y de interrelación difícil de superar.

De este modo, se entiende que una vez que la mujer se realiza la cirugía de mastectomía, debe tener un cuidado especial, pues, existen complicaciones postoperatorias significativas, entre las cuales se encuentran: infección de la herida, seroma, neumotórax, necrosis de tejido, hemorragia entre otras. De allí, la importancia, que debe darle la mujer a tener una atención adecuada para evitar estas consecuencias y lograr su respectiva recuperación de manera favorable. Es importante destacar que, cada uno de los planteamientos citados, son relevantes para la conducción del presente artículo, pues, logran insertar de manera coherente nuevas ideas, para llegar a un análisis en cuanto a las complicaciones que presentan las pacientes de cáncer de mama sometidas a una mastectomía.

\section{Método.}

El proceso investigativo, para darle correspondencia al propósito previamente establecido, lleva a considerar pertinente la ubicación del método como herramienta esencial para adecuar los contenidos referidos al tratamiento del dolor postquirúrgico por vía epidural y en función de las 


\section{Complicaciones postoperatorias a las pacientes sometidas a una mastectomía}

Vol. 3, núm. 4., (2019)

Gabriela Cecilia Arroyo Calvopiña; Anthony James Chávez Salazar; Jessica Fernanda Toledo Cascante; Silvia Jimena Zambrano Salvatierra; Fabricio Wladimir Gutiérrez Rivera; Michelle

Madeleyne Salazar Bustamante

diferentes apreciaciones se selecciona el método que hace posible redimensionar los eventos encargados de estructurar la recopilación de la información necesaria para la respectiva sustentación del tema seleccionado.

Como método para la investigación se utiliza el análisis e interpretación de datos académicos, información y diferentes conceptos para orientar en forma precisa una posición, así mismo Mata (2018) define al método como: "una estrategia con la que se observa y reflexiona sistemáticamente sobre realidades teóricas y empíricas usando para ello diferentes tipos de documentos donde se indaga, interpreta, presenta datos e información sobre un tema determinado" (p.22). De lo anterior citado, se puede reflexionar que la interpretación de diferentes realidades es necesaria para lograr presentar información orientada en forma correcta y académica, dándole sentido coherente a la investigación.

Por ello, es importante ubicar este artículo en un campo de las ciencias sociales, pues, su relación con un campo metodológico, se convierte en la fuente esencial para encaminar el hecho investigativo, dentro de un campo científico particular, que hace posible responder a las necesidades de indagar. De allí, que se consideró pertinente el método deductivo, dicha selección hizo posible, desglosar los contenidos de forma general, para luego elaborar las apreciaciones que sirvieron de base en la construcción definitiva del artículo.

\section{Tipo de Investigación.}

Desde el punto de vista metodológico es una investigación documental, donde se utiliza herramientas para conocer principios y procedimientos que permitan desarrollar la información y el conocimiento. Según Suárez de Paredes (2010)), la investigación documental "es aquella búsqueda o indagación que se basa en la localización, registro, recuperación, análisis e interpretación de fuentes bibliográficas, hemorográficas, así como fuentes de carácter primario básico" (p.15). Es decir, para concretar esta investigación documental, se puede indicar que la misma fue realizada cumpliendo la metodología del método científico, al interpretar y analizar informaciones de documentos académicos vinculados al tema para logra en forma coherente un resultado que apoye y relacione el contenido en función de las necesidades de la investigación. 


\section{Complicaciones postoperatorias a las pacientes sometidas a una mastectomía}

Vol. 3, núm. 4., (2019)

Gabriela Cecilia Arroyo Calvopiña; Anthony James Chávez Salazar; Jessica Fernanda Toledo Cascante; Silvia Jimena Zambrano Salvatierra; Fabricio Wladimir Gutiérrez Rivera; Michelle Madeleyne Salazar Bustamante

\section{Fuentes Documentales.}

La interpretación de las fuentes informativas de acuerdo a lo requerido en la investigación académica se lleva a incluir los diferentes datos, documentos existentes para generar un orden de ideas adecuadas al tema. Para Angulo (2017) las fuentes documentales "son los documentos, sucesos u objetos que trasmiten información significativa sobre el origen de la información." (p.36). De acuerdo con lo citado, se entiende que, mediante una adecuada selección de las técnicas documentales, el investigador, logra primero organizar las actividades vinculadas con la lectura rápida, para luego concretar el trabajo bajo una dirección de interpretaciones generales en pro de lograr la respectiva construcción del contenido.

En consecuencia, la realización de este artículo se cumplió mediante la utilización de la lectura e interpretación de documentos relacionados al tema, para generar una matriz nueva de opinión en relación al tema abordado. En otras palabras, se precisaron aquellos aportes teóricos fundamentales para la construcción del cuerpo general de la investigación y en pro de ello, garantizar así de manera eficiente el método previamente seleccionado.

\section{Técnicas para la Recolección de la Información.}

La recolección de datos en una investigación documental lleva a una estrecha diversidad de técnicas y herramientas para ser utilizadas por el investigador. Logrando centrarse en la lectura de documentos académicos, estadísticas y datos recolectados que permiten realizar un análisis profundo e interpretativo de todos los documentos. Ángulo (ob.cit) la recolección de información "se refiere al uso de una gran diversidad de técnicas y herramientas que pueden ser utilizadas por el analista para desarrollar los sistemas de información” (p.52). Al interpretar lo antes expuesto, se puede decir que el proceso de recolección de información en el desarrollo de la investigación, se cumplió mediante el procesamiento de la temática en forma correcta.

En consecuencia, para el desarrollo de este apartado, se precisó la utilización de la lectura reflexiva, como herramienta que ayuda al investigador a valorar de forma coherente el contenido del trabajo, con el fin de ofrecerlo de manera precisa y cuya pertinencia permita no sólo comprender su 


\section{Complicaciones postoperatorias a las pacientes sometidas a una mastectomía}

Vol. 3, núm. 4., (2019)

Gabriela Cecilia Arroyo Calvopiña; Anthony James Chávez Salazar; Jessica Fernanda Toledo Cascante; Silvia Jimena Zambrano Salvatierra; Fabricio Wladimir Gutiérrez Rivera; Michelle

Madeleyne Salazar Bustamante

contenido, sino dispones de diferentes posibilidades para traspolar sus consideraciones desde diferentes perspectivas.

\section{Resultados.}

Para la continuidad del proceso de interpretación y recolección de información, se hace necesario llegar a la organización de los diferentes contenidos de manera particular, para así afianzar diferentes consideraciones generales que permitan precisar los argumentos desde una visión global. Por lo tanto, los aspectos funcionales referidos al tema fueron desglosados de la siguiente manera:

\section{Cáncer de Mama.}

El cáncer de mama es el crecimiento descontrolado de las células mamarias. Es el resultado de mutaciones, o cambios anómalos, en los genes que regulan el crecimiento de las células y las mantienen sanas. Los genes se encuentran en el núcleo de las células, el cual actúa como la sala de control de cada célula. Normalmente, las células del cuerpo se renuevan mediante un proceso específico llamado crecimiento celular: las células nuevas y sanas ocupan el lugar de las células viejas que mueren. Pero con el paso del tiempo, las mutaciones pueden activar ciertos genes y desactivar otros en una célula. La célula modificada adquiere la capacidad de dividirse sin ningún tipo de control u orden, por lo que produce más células iguales y genera un tumor.

Un tumor puede ser benigno (no es peligroso para la salud) o maligno (es potencialmente peligroso). Los tumores benignos no son considerados cancerosos: sus células tienen una apariencia casi normal, crecen lentamente y no invaden tejidos próximos ni se propagan hacia otras partes del cuerpo. Los tumores malignos son cancerosos. De no ser controladas, las células malignas pueden propagarse más allá del tumor original hacia otras partes del cuerpo. Por ello, el término cáncer de mama hace referencia a un tumor maligno que se ha desarrollado a partir de células mamarias. Generalmente, el cáncer de mama se origina en las células de los lobulillos, que son las glándulas productoras de leche, o en los conductos, que son las vías que transportan la leche desde los lobulillos hasta el pezón. Con menos frecuencia, el cáncer de mama puede 


\section{Complicaciones postoperatorias a las pacientes sometidas a una mastectomía}

Vol. 3, núm. 4., (2019)

Gabriela Cecilia Arroyo Calvopiña; Anthony James Chávez Salazar; Jessica Fernanda Toledo Cascante; Silvia Jimena Zambrano Salvatierra; Fabricio Wladimir Gutiérrez Rivera; Michelle Madeleyne Salazar Bustamante

originarse en los tejidos estromales, que incluyen a los tejidos conjuntivos grasos y fibrosos de la mama.

En este sentido, Casillas (2016), es el más común en mujeres de todo el mundo, representa el 16\% del total de casos por neoplasias. Además, cada año se detectan 1.38 millones de nuevos casos y se registran 458 mil muertes. Este tipo de cáncer es una de las enfermedades que no distingue a la población de los países desarrollados y en vías de desarrollo. En el 2012, destaca el autor citado, que en las Américas 462 mil mujeres fueron diagnosticadas y 100 mil fallecieron por esta enfermedad. También, se estima que para el año 2030, los nuevos casos aumenten un 34\%.

En esta misma dirección, se puede resaltar los aportes dados por Chavira y Pérez (2019), quienes indicaron en relación a la media de edad fue de $54 \pm 12$ con un mínimo de 33 y un máximo de 85 años, además el $48 \%$ tenía más de 50 años; por otro lado, el 50\% tenía una ocupación remunerada durante su enfermedad; $67 \%$ contaba con pareja; $23 \%$ con nivel educativo básico (primaria y secundaria) y sólo el 13\% con nivel superior. En lo que respecta a su religión, 46\% son católicas y el 54\% habitaba en la Ciudad de México.

En cuanto a los aspectos de la mujer y su historia médica, el $12 \%$ contaba con antecedentes heredofamiliares de cáncer de mama, Respecto a sus antecedentes personales patológicos, el 54\% tenía otra enfermedad agregada al cáncer; 23\% diabetes mellitus, 35\% hipertensión arterial y 8\% con enfermedad vascular periférica. También, el $48 \%$ tomaba algún medicamento y el 35\% contaba con antecedentes no patológicos, de las cuales el $27 \%$ fumó alguna vez en su vida y $15 \%$ tomaba bebidas alcohólicas ocasionalmente.

Al 67\% de las mujeres se le diagnosticó cáncer con biopsia tipo incisional. El 90\% con cáncer tipo ductal, encontrándose estadios ECII con 39\% y ECIII con 35\%. En cuanto a la lateralidad de la cirugía, el $62 \%$ fue de lado izquierdo y la cirugía que más se realiza es la mastectomía radical modificada con el 67\%. Al 92\% de las mujeres se les retiraron ganglios linfáticos con una media de $11 \pm 5$. 


\section{Complicaciones postoperatorias a las pacientes sometidas a una mastectomía}

Vol. 3, núm. 4., (2019)

Gabriela Cecilia Arroyo Calvopiña; Anthony James Chávez Salazar; Jessica Fernanda Toledo Cascante; Silvia Jimena Zambrano Salvatierra; Fabricio Wladimir Gutiérrez Rivera; Michelle Madeleyne Salazar Bustamante

\section{Figura $\mathbf{N}^{\circ} 1$ Anatomía de la Mama}

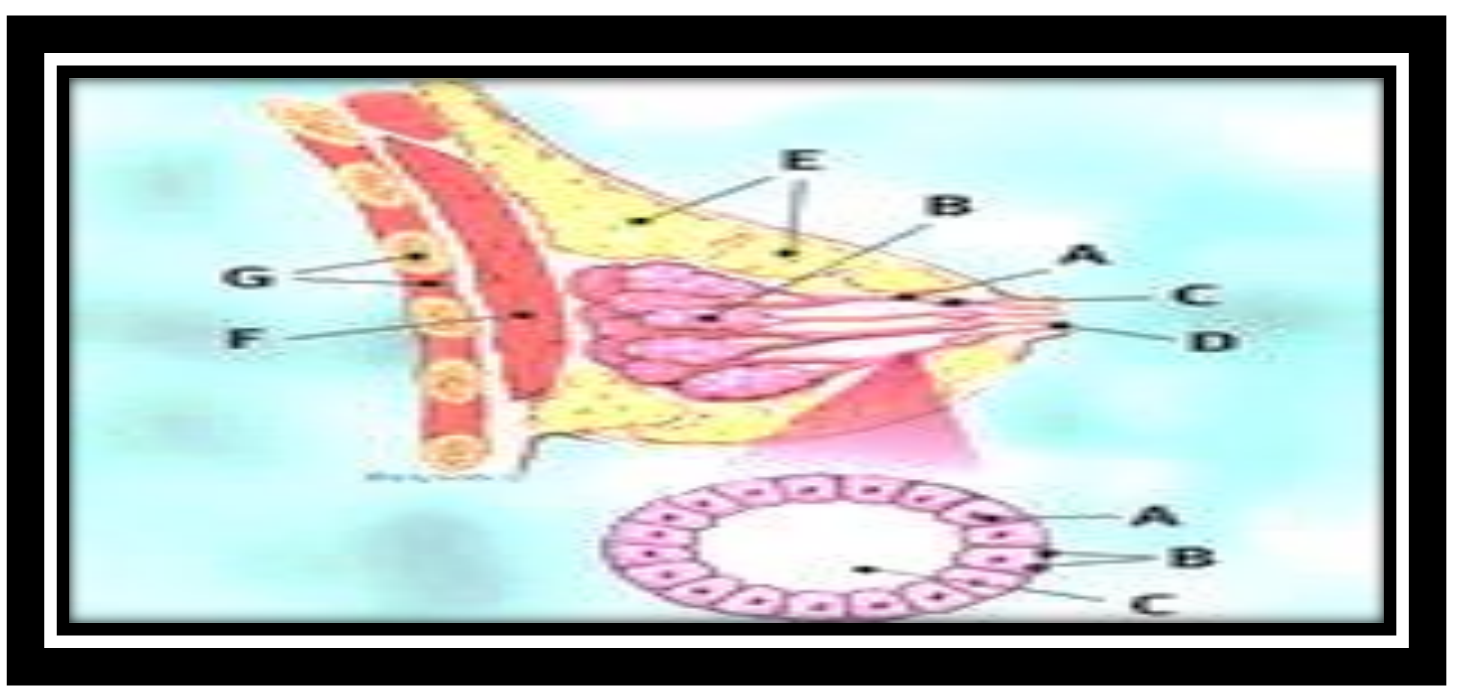

Fuente: Casillas (ob.cit)

Con el paso del tiempo, las células cancerígenas pueden invadir el tejido mamario sano circundante y llegar a los ganglios linfáticos (pequeños órganos que eliminan sustancias extrañas del cuerpo) de las axilas. Si las células cancerígenas llegan a los ganglios linfáticos, obtienen una puerta de acceso hacia otras partes del cuerpo. Los estadios del cáncer de mama hacen referencia a lo lejos que se han propagado las células cancerígenas más allá del tumor original. El cáncer de mama siempre se origina por una anomalía genética (un error en el material genético). No obstante, solo un 5-10\% de los casos son producto de una anomalía heredada de la madre o el padre. En cambio, el 85-90\% de los casos de cáncer de mama tienen su origen en anomalías genéticas vinculadas al proceso de envejecimiento y el desgaste natural de la vida.

Existen pasos que toda persona puede seguir para mantener su cuerpo lo más sano posible, como seguir una dieta balanceada, mantener un peso saludable, no fumar, limitar el consumo de alcohol y ejercitarse de forma regular (aprende lo que puedes hacer para manejar los factores de riesgos del cáncer de mama. Aunque pueden tener algún impacto en el riesgo de padecer cáncer de mama, no pueden eliminar el riesgo. En esta dirección, Casillas (ob.cit), especifica las condiciones generales de los diferentes estadios del cáncer de mama. 


\section{Complicaciones postoperatorias a las pacientes sometidas a una mastectomía}

Vol. 3, núm. 4., (2019)

Gabriela Cecilia Arroyo Calvopiña; Anthony James Chávez Salazar; Jessica Fernanda Toledo Cascante; Silvia Jimena Zambrano Salvatierra; Fabricio Wladimir Gutiérrez Rivera; Michelle Madeleyne Salazar Bustamante

Estadio 0: se utiliza para describir los casos de cáncer de mama no invasivos, como el CDIS (carcinoma ductal in situ). En el estadio 0, no hay indicios de células cancerígenas o células anómalas no cancerosas que salen de la zona de la mama en la que se originaron o que toman o invaden tejidos normales circundantes.

Estadio I: describe el cáncer de mama invasivo (las células cancerígenas toman o invaden el tejido mamario normal que las rodea). El estadio I está dividido en subcategorías, conocidas como IA e IB. En cuanto al estadio IA describe el cáncer de mama invasivo en el que: el tumor mide hasta $2 \mathrm{~cm}$ Y el cáncer no se ha extendido más allá de la mama; no hay ganglios linfáticos afectados. El estadio IB describe el cáncer de mama invasivo en el que: no hay tumor en la mama; en cambio, se observan en los ganglios linfáticos pequeños grupos de células cancerígenas superiores a $0,2 \mathrm{~mm}$, pero inferiores a $2 \mathrm{~mm}$. Se observa un tumor en la mama inferior a $2 \mathrm{~cm}$ y pequeños grupos de células cancerígenas superiores a $0,2 \mathrm{~mm}$, pero inferiores a $2 \mathrm{~mm}$ en los ganglios linfáticos. En el cáncer de mama de estadio I, es posible la invasión microscópica. Esto significa que las células cancerígenas recién han comenzado a invadir el tejido ubicado en las paredes del conducto o el lobulillo, pero estas células cancerígenas invasoras no miden más de $1 \mathrm{~mm}$.

Estadio II: Se divide en las subcategorías IIA y IIB. El estadio IIA describe el cáncer de mama invasivo en el que no hay ningún tumor en la mama, pero se detectan células cancerígenas (que superan los $2 \mathrm{~mm}$ ) en 1-3 ganglios linfáticos axilares (debajo del brazo) o en los ganglios linfáticos cerca del esternón (encontrado durante una biopsia del ganglio centinela). El tumor mide $2 \mathrm{~cm}$ o menos, y se ha propagado hacia los ganglios linfáticos axilares. El tumor mide entre 2 y $5 \mathrm{~cm}$, y no se ha propagado hacia los ganglios linfáticos axilares. El estadio IIB describe el cáncer de mama invasivo en el que: el tumor mide entre 2 y $5 \mathrm{~cm}$, y se observan en los ganglios linfáticos pequeños grupos de células cancerígenas superiores a $0,2 \mathrm{~mm}$, pero inferiores a $2 \mathrm{~mm}$, el tumor mide entre 2 y $5 \mathrm{~cm}$, y el cáncer se ha propagado en 1-3 ganglios linfáticos axilares o en los ganglios linfáticos cercanos al esternón (encontrado durante una biopsia del ganglio centinela) el tumor mide más de $5 \mathrm{~cm}$, pero no se ha propagado hacia los ganglios linfáticos axilares. 


\section{Complicaciones postoperatorias a las pacientes sometidas a una mastectomía}

Vol. 3, núm. 4., (2019)

Gabriela Cecilia Arroyo Calvopiña; Anthony James Chávez Salazar; Jessica Fernanda Toledo Cascante; Silvia Jimena Zambrano Salvatierra; Fabricio Wladimir Gutiérrez Rivera; Michelle

Madeleyne Salazar Bustamante

El estadio III se divide en las subcategorías IIIA, IIIB y IIIC. El estadio IIIA describe el cáncer de mama invasivo en el que: no hay tumor en la mama o el tumor puede tener cualquier tamaño, y se detectó la presencia de cáncer en 4-9 ganglios linfáticos axilares o en los ganglios linfáticos cercanos al esternón (encontrado durante estudios de imágenes o una exploración física) el tumor mide más de $5 \mathrm{~cm}$, y se observan en los ganglios linfáticos pequeños grupos de células cancerígenas superiores a $0,2 \mathrm{~mm}$, pero inferiores a $2 \mathrm{~mm}$, el tumor mide más de $5 \mathrm{~cm}$, y el cáncer se ha propagado en 1-3 ganglios linfáticos axilares o en los ganglios linfáticos cercanos al esternón (encontrado durante una biopsia del ganglio centinela).

El estadio IIIB describe el cáncer de mama invasivo en el que: el tumor tiene un tamaño indefinido y se ha propagado hacia la pared torácica o la piel de la mama, y ha provocado inflamación o una úlcera $\mathrm{Y}$ pudo haberse propagado hacia 9 ganglios linfáticos axilares pudo haberse propagado hacia los ganglios linfáticos cercanos al esternón El cáncer de mama inflamatorio pertenece por lo menos al estadio IIIB. Las características típicas del cáncer de mama inflamatorio son: enrojecimiento de un gran sector de la piel de la mama, la mama se siente caliente y puede estar inflamada las células cancerígenas se propagan hacia los ganglios linfáticos y pueden encontrarse en la piel.

Mientras que el estadio IIIC describe el cáncer de mama invasivo en el que: puede que no haya indicios de la enfermedad en la mama o, si hay un tumor, puede tener cualquier tamaño y haberse propagado hacia la pared torácica o a la piel de la mama el cáncer se ha propagado en 10 o más ganglios linfáticos axilares, el cáncer se ha propagado hacia los ganglios linfáticos ubicados sobre o debajo de la clavícula el cáncer se ha propagado hacia los ganglios linfáticos axilares o los ganglios linfáticos cercanos al esternón.

Estadio IV: describe el cáncer de mama invasivo que se ha propagado más allá de la mama y los ganglios linfáticos circundantes hacia otros órganos del cuerpo, como los pulmones, ganglios linfáticos distantes, la piel, los huesos, el hígado y el cerebro. Tal vez exista metástasis para describir el cáncer de mama de estadio IV. Un cáncer puede ser de estadio IV en un primer 


\section{Complicaciones postoperatorias a las pacientes sometidas a una mastectomía}

Vol. 3, núm. 4., (2019)

Gabriela Cecilia Arroyo Calvopiña; Anthony James Chávez Salazar; Jessica Fernanda Toledo Cascante; Silvia Jimena Zambrano Salvatierra; Fabricio Wladimir Gutiérrez Rivera; Michelle Madeleyne Salazar Bustamante

diagnóstico o puede tratarse de una recurrencia de un cáncer de mama anterior que se ha propagado hacia otras partes del cuerpo.

Cada uno de los estadios antes referidos, llevan a precisar que la evolución del tumor que aparece en la mama, presenta diferentes cambios y medidas, aspectos determinantes para llegar a estimar la cirugía como alternativa para erradicar los ganglios linfáticos axilares, como resultado al desarrollo que el tumor ha logrado alcanzar o se encuentre propagado en la mujer. Ante, esto, el tratamiento de abordaje se encuentra determinado por la realización de una mastectomía.

\section{Complicaciones Postoperatorias en Pacientes con Mastectomía.}

La mastectomía es una manera de tratar el cáncer de seno mediante la extirpación quirúrgica de todo el seno. A menudo se hace cuando una mujer no puede ser tratada con cirugía con conservación del seno tumorectomía, la cual preserva la mayor parte del seno. También se puede hacer si una mujer opta por la mastectomía en lugar de la cirugía con conservación del seno por motivos personales. Las mujeres con un riesgo muy alto de padecer otro cáncer a veces se someten a una mastectomía doble, la extirpación de ambos senos. Hay varios tipos diferentes de mastectomías, en función de cómo se realiza la cirugía y cuánto tejido se extrae, las cuales son referidas por Chavira y Pérez (ob.cit)

Mastectomía simple (o total): En este procedimiento, el cirujano extirpa todo el seno, incluyendo el pezón, la areola y la piel. Dependiendo de la situación, puede o no ser necesario extraer algunos ganglios linfáticos de la axila. La mayoría de las mujeres, si son hospitalizadas, pueden ser dadas de alta al día siguiente.

Mastectomía con conservación de piel: En este procedimiento, se deja intacta la mayor parte de la piel sobre el seno. Se extirpa solamente el tejido mamario, el pezón y areola. La cantidad de tejido mamario extirpado es la misma que en la mastectomía simple. Los implantes o el tejido de otras partes del cuerpo se usan al momento de la cirugía para reconstruir el seno. Muchas mujeres prefieren la mastectomía con preservación de piel porque ofrece la ventaja de que deja menos tejido cicatricial y un seno reconstruido con una apariencia más natural. Sin embargo, puede que 


\section{Complicaciones postoperatorias a las pacientes sometidas a una mastectomía}

Vol. 3, núm. 4., (2019)

Gabriela Cecilia Arroyo Calvopiña; Anthony James Chávez Salazar; Jessica Fernanda Toledo Cascante; Silvia Jimena Zambrano Salvatierra; Fabricio Wladimir Gutiérrez Rivera; Michelle

Madeleyne Salazar Bustamante

no sea apropiada para tumores más grandes o para aquellos que están cerca de la superficie de la piel. El riesgo de recurrencia local del cáncer con este tipo de mastectomía es igual que con otros tipos de mastectomías.

Mastectomía con conservación del pezón: La mastectomía con conservación del pezón es una variación de la mastectomía con conservación de piel. En este procedimiento, se extirpa el tejido del seno, pero la piel y el pezón del seno se dejan en su lugar. Después de este procedimiento se puede realizar una reconstrucción del seno. Durante el procedimiento, el cirujano a menudo extirpa el tejido del seno que está debajo del pezón (y areola) para determinar si contiene células cancerosas. Si se encuentra cáncer en este tejido, el pezón tiene que ser extirpado. Aun cuando no se encuentre cáncer debajo del pezón, algunos médicos administran una dosis de radiación al tejido del pezón durante o después de la cirugía para tratar de reducir el riesgo de que regrese el cáncer.

Este procedimiento es una opción más frecuente en mujeres que padecen un cáncer pequeño en etapa temprana cerca de la parte externa del seno, sin ningún signo de cáncer en la piel o cerca del pezón. Si el tumor mamario es más grande o se encuentra cerca al pezón, hay más probabilidades de que las células cancerosas se escondan en el pezón, lo que representa un mayor riesgo de que regrese el cáncer si no se extrae el pezón.

Sin embargo, existen algunas complicaciones con las cirugías para la conservación del pezón. Después de esta operación, es posible que el pezón no tenga un buen suministro de sangre, lo que puede atrofiar o deformar el tejido. Debido a que también se cortan los nervios, a menudo queda poca o ninguna sensación en el pezón. En mujeres con senos más grandes, el pezón puede lucir fuera de lugar después de reconstruir el seno. Como resultado, muchos médicos creen que esta cirugía es más eficaz en mujeres con senos pequeños o medianos. Este procedimiento deja poca cicatriz visible, pero si no se realiza adecuadamente, puede dejar más tejido del seno que las otras formas de mastectomía. Esto podría causar más riesgo de padecer cáncer que si se realiza una mastectomía simple o con preservación de piel. En el pasado, esto representaba más un problema, pero las mejoras en la técnica han contribuido a que esta cirugía sea más segura. Aun así, muchos 


\section{Complicaciones postoperatorias a las pacientes sometidas a una mastectomía}

Vol. 3, núm. 4., (2019)

Gabriela Cecilia Arroyo Calvopiña; Anthony James Chávez Salazar; Jessica Fernanda Toledo Cascante; Silvia Jimena Zambrano Salvatierra; Fabricio Wladimir Gutiérrez Rivera; Michelle Madeleyne Salazar Bustamante

expertos no consideran que la mastectomía con conservación del pezón sea un tratamiento convencional para el cáncer de seno.

Mastectomía Radical Modificada: Una mastectomía radical modificada combina una mastectomía simple con la extirpación de los ganglios linfáticos axilares (disección de los ganglios linfáticos axilares).

Mastectomía Radical: En esta operación extensa, el cirujano extirpa todo el seno, los ganglios linfáticos axilares y los músculos pectorales (pared torácica) que se encuentran debajo del seno. Esta cirugía fue muy común en el pasado, pero se ha descubierto que una cirugía menos extensa (como la mastectomía radical modificada) ofrece los mismos resultados con menos efectos secundarios. Por lo tanto, hoy día esta cirugía se realiza en pocas ocasiones. Puede que esta operación se siga haciendo para tumores grandes que están invadiendo los músculos pectorales.

Mastectomía Doble: Si se realiza una mastectomía en ambos senos, se denomina mastectomía doble (o bilateral). Cuando se realiza este procedimiento, a menudo se lleva a cabo como cirugía de reducción de riesgo en las mujeres con un riesgo muy alto de padecer cáncer en sus senos, como aquellas con una mutación en el gen BRCA. La mayoría de estas mastectomías son simples, pero algunas podrían preservar el pezón. 


\section{Complicaciones postoperatorias a las pacientes sometidas a una mastectomía}

Vol. 3, núm. 4., (2019)

Gabriela Cecilia Arroyo Calvopiña; Anthony James Chávez Salazar; Jessica Fernanda Toledo Cascante; Silvia Jimena Zambrano Salvatierra; Fabricio Wladimir Gutiérrez Rivera; Michelle Madeleyne Salazar Bustamante

\section{Figura $\mathbf{N}^{\circ} 2$ Mastectomía}

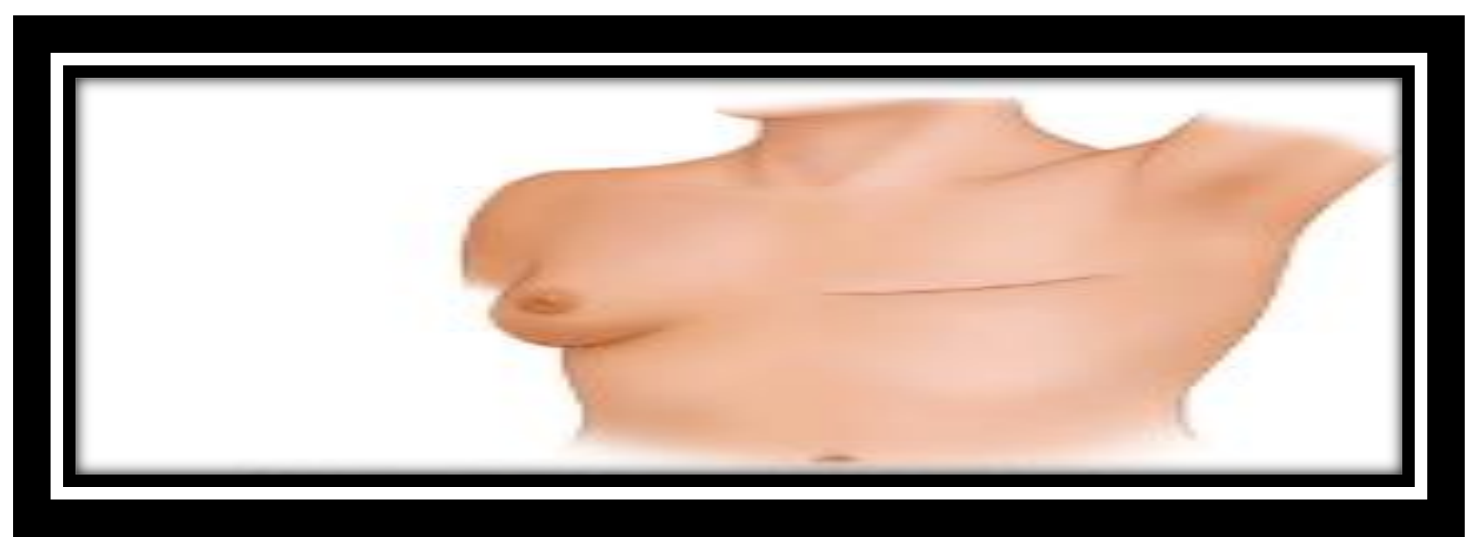

Fuente: Chaivra y Pérez (ob.cit)

Muchas mujeres que padecen cáncer en etapas tempranas pueden elegir entre la cirugía con conservación del seno y la mastectomía. Es posible que usted se incline inicialmente por la mastectomía para deshacerse del cáncer lo más rápido posible. Sin embargo, la realidad es que la mastectomía no provee una mejor probabilidad de una supervivencia a largo plazo o un mejor resultado del tratamiento en la mayoría de los casos. Los estudios que siguen a miles de mujeres por más de 20 años muestran que cuando se puede hacer la cirugía con conservación del seno y administrar radiación, en lugar de la mastectomía, ésta última no provee una mejor probabilidad de supervivencia del cáncer de seno. 


\section{Complicaciones postoperatorias a las pacientes sometidas a una mastectomía}

Vol. 3, núm. 4., (2019)

Gabriela Cecilia Arroyo Calvopiña; Anthony James Chávez Salazar; Jessica Fernanda Toledo Cascante; Silvia Jimena Zambrano Salvatierra; Fabricio Wladimir Gutiérrez Rivera; Michelle Madeleyne Salazar Bustamante

Al hacer referencia a las complicaciones postoperatorias, es importante destacar que, una vez realizada la mastectomía, puede aparecer estas complicaciones, las cuales se determinan por un carácter multifactorial, ya que influyen aspectos inherentes a la enfermedad misma, el tipo de tratamientos que se ofrecen y las características individuales de las mujeres. Al respecto, Jiang (2016), precisa que entre las complicaciones postoperatorias se resaltan la rehospitalización dentro de los 30 días; complicaciones de la herida dentro de los primeros 2 años postoperatorios; e infecciones de piel o tejidos blandos dentro de los primeros 2 años postoperatorios.

Asimismo, argumenta que el $67 \%$ de las mujeres se le diagnosticó cáncer con biopsia tipo incisional. El 90\% con cáncer tipo ductal, encontrándose estadios ECII con 39\% y ECIII con 35\%. En cuanto a la lateralidad de la cirugía, el $62 \%$ fue de lado izquierdo y la cirugía que más se realiza es la mastectomía radical modificada con el 67\%. Al 92\% de las mujeres se les retiraron ganglios linfáticos con una media de $11 \pm 5$. El $42 \%$ de las mujeres presentaron alguna complicación, el $23 \%$ presentó algún tipo de dolor (hombro, brazo y/o zona de la cirugía), el 4\% tuvo seroma con una media de días de $145 \pm 120$; además el $23 \%$ se complicó por infección con una media de $40 \pm$ $32 ; 27 \%$ por dehiscencia de herida con $30 \pm 9$ días después de su egreso; y el 23\% por linfedema con una media de $181 \pm 81$ días. 


\section{Complicaciones postoperatorias a las pacientes sometidas a una mastectomía}

Vol. 3, núm. 4., (2019)

Gabriela Cecilia Arroyo Calvopiña; Anthony James Chávez Salazar; Jessica Fernanda Toledo Cascante; Silvia Jimena Zambrano Salvatierra; Fabricio Wladimir Gutiérrez Rivera; Michelle Madeleyne Salazar Bustamante

\section{Gráfico $\mathbf{N}^{\circ} 1$ Complicaciones Postoperatorias por Mastectomía}

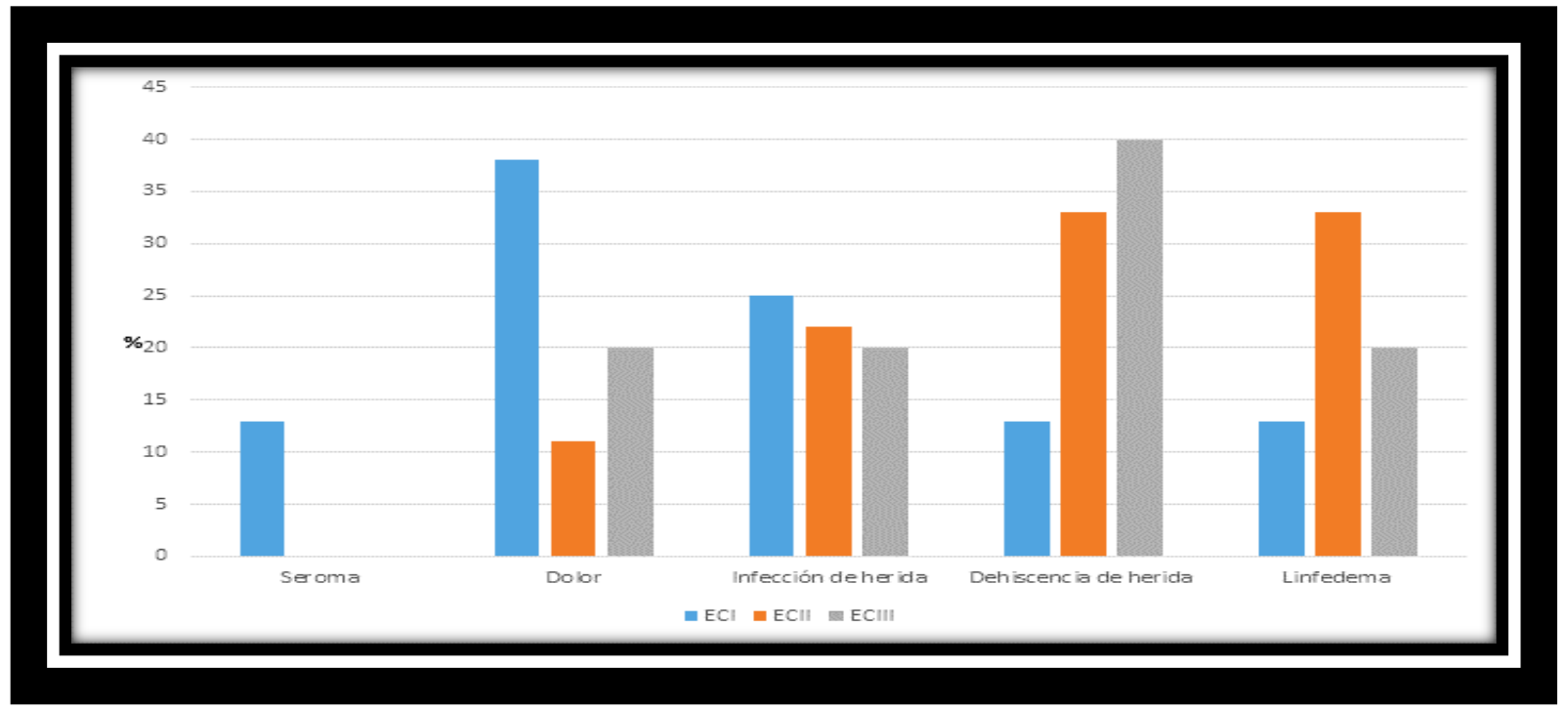

Fuente: Jiang (ob.cit)

La gráfica $\mathrm{n}^{\circ} 1$ las mujeres con ECI presentaron en mayor proporción dolor (38\%), en estadio ECII se complican más por linfedema y dehiscencia de herida (33\% en ambos casos) y en estadio ECIII prevalece en mayor proporción la dehiscencia de herida (40\%). Además, a las mujeres que se les realizó mastectomía radical modificada, mostraron mayor proporción de complicaciones (77\%) comparado con cuadrantectomía (5\%) y mastectomía total (18\%).

Con relación a la infección de herida, la literatura reporta una prevalencia del uno al $20 \%$ y refieren que uno de los microorganismos que la ocasiona es el Staphylococcus aureus parte de la flora cutánea, así como de otros factores de la mujer como la edad, obesidad, y diabetes mellitus. Además, el 23\% fue catalogado como complicación temprana, ya que su aparición va desde los 17 días después de haber egresado del hospital. Por ello, es necesario reestructurar los procesos de cuidados pre, trans, postoperatorios, así como la educación a la paciente y su familia para el manejo de sus heridas en el hogar, a fin de evitar este tipo de complicación, por ejemplo: la higiene de manos intrahospitalario y en el hogar, curación de herida, uso de antibioticoterapia entre otros. 


\section{Complicaciones postoperatorias a las pacientes sometidas a una mastectomía}

Vol. 3, núm. 4., (2019)

Gabriela Cecilia Arroyo Calvopiña; Anthony James Chávez Salazar; Jessica Fernanda Toledo Cascante; Silvia Jimena Zambrano Salvatierra; Fabricio Wladimir Gutiérrez Rivera; Michelle Madeleyne Salazar Bustamante

En cuanto al seroma se presentó en el $4 \%$ de los casos estudiados, inferior a lo que evidencia la literatura, ya que se ha reportado en otros contextos una incidencia de hasta un $30 \%$. Algunos factores asociados son bajos niveles de fibrinógeno de la paciente, otro es el uso inadecuado de drenajes externos. Estudios han reportado que el uso de drenajes cerrados prolongados vs a corto plazo vs sin drenaje no reduce la tasa de seroma. Algunos han optado por la inmovilización del hombro, pero se menciona que esta intervención podría aumentar el riesgo de linfedema. La recomendación es realizar fisioterapia temprana, para contribuir a la reducción de esta complicación.

Otra complicación presentada en este estudio es el dolor que en su mayoría es de tipo neuropático, con una prevalencia del $23 \%$, comparado con la literatura que reporta entre 20 y $50 \%$ de los casos, pero se refiere que los factores son en su mayoría inciertos, sin embargo, entre ellos se han identificado la edad, los tumores grandes, la quimioterapia, la radioterapia y depresión, además que la etiología es de carácter neuropático y que algunas de las estrategias eficaces son el uso de fármacos antidepresivos. Esta complicación es de esperarse en mujeres diagnosticadas en estadios avanzados por lo que reciben tratamiento multimodal (cirugía, quimioterapia, radioterapia y hormonoterapia).

Por último, el linfedema, complicación derivada del retiro de ganglios linfáticos con un $23 \%$ y reportado por otros estudios con porcentajes que oscilan del cinco al 66\%. Esta complicación está completamente relacionada con la extracción de ganglios linfáticos y la radioterapia como parte del tratamiento adyuvante. Otros factores están relacionados con las características de la mujer como la obesidad, el tabaquismo, entre otros. Algunas de las recomendaciones para reducir el riesgo de linfedema están centradas en un manejo interdisciplinario de manera preventiva y correctiva; como es el uso de método ganglio centinela para reducir en gran medida el número de ganglios a extirpar, así como el uso de manga de compresión y rehabilitación temprana del brazo afectado. 


\section{Complicaciones postoperatorias a las pacientes sometidas a una mastectomía}

Vol. 3, núm. 4., (2019)

Gabriela Cecilia Arroyo Calvopiña; Anthony James Chávez Salazar; Jessica Fernanda Toledo Cascante; Silvia Jimena Zambrano Salvatierra; Fabricio Wladimir Gutiérrez Rivera; Michelle

Madeleyne Salazar Bustamante

Neumotórax: Es rara complicación, se desarrolla cuando el cirujano perfora la pleura parietal con la disección del tejido extendida o con los intentos de hemostasia para los perforadores de la musculatura intercostal. El neumotórax es más común en los pacientes que se someten a una mastectomía radical.

La necrosis de tejido: Una complicación común de la cirugía de mama es la necrosis de los colgajos de piel desarrollados o en los márgenes de la piel.

Hemorragia: es una complicación post-operatoria en el $1 \%$ a $4 \%$ de los pacientes y se manifiesta por la inflamación excesiva de la zona quirúrgica. El reconocimiento precoz de esta complicación es imprescindible.

De este modo, se puede complementar que la realización de una cirugía como la mastectomía, no es más que un procedimiento quirúrgico que consiste en la extirpación de parte o de toda la mama, gracias a ello se ha observado mejoras en los métodos de tratamiento oncológico que han posibilitado una considerable disminución de la tasa de mortalidad de la enfermedad y en la actualidad, alrededor del $83 \%$ de las mujeres con cáncer de mama sobreviven a los 5 años

En consecuencia, cuando la mujer es sometida a un proceso quirúrgico de mastectomía, es importante mantener vigilancia y control postoperatorio, para ello, se hace necesario que el cirujano indique algunas recomendaciones entre ellas:

Medicamentos para el alivio del dolor: el cirujano indicará algún medicamento para lograr el alivio del dolor una vez dada la salida del hospital.

Cuidado del Vendaje (o apósito) en el sitio de la incisión: Es posible que el cirujano indique no quitarse el vendaje, sino que esperar hasta la primera visita de seguimiento para retirarlo.

Cuidado del Tubo de Drenado Quirúrgico: al tener un tubo de drenado en el área del pecho o la axila, debe retirarse antes de salir de alta. Sin embargo, algunas veces es necesario dejarlo hasta la primera consulta de seguimiento con el médico, que suele ser una o dos semanas después de la cirugía. Es, importante vaciar el líquido del recipiente de drenaje unas veces por día. 


\section{Complicaciones postoperatorias a las pacientes sometidas a una mastectomía}

Vol. 3, núm. 4., (2019)

Gabriela Cecilia Arroyo Calvopiña; Anthony James Chávez Salazar; Jessica Fernanda Toledo Cascante; Silvia Jimena Zambrano Salvatierra; Fabricio Wladimir Gutiérrez Rivera; Michelle Madeleyne Salazar Bustamante

Puntos de sutura y grapas: la mayoría de los cirujanos usan suturas (puntos) que se disuelven con el tiempo, de modo que ya no es necesario quitarlas. Las grapas quirúrgicas, otra forma de cerrar la herida, se quitan durante la primera consulta posterior a la cirugía.

Reconocimiento de indicios de infección: el cirujano debe explicar qué hacer si se presenta una infección en la incisión y cuándo debes llamar al consultorio.

Ejercicios para el brazo: es posible que el cirujano o la enfermera indiquen una rutina de ejercicios que pueden realizar para evitar la rigidez del brazo y el hombro del lado en que te realizaron la cirugía. Generalmente se comenzará a practicar los ejercicios la mañana siguiente a la cirugía. Algunos se deben evitar hasta que se quite el tubo de drenado. El cirujano también debe proporcionarte instrucciones escritas y detalladas sobre cómo hacer los ejercicios.

Reconocimiento de indicios de linfedema: al realizar una disección axilar, el especialista debe explicar cómo cuidar el brazo y estar alerta para detectar indicios de linfedema.

\section{Conclusiones.}

Cada uno de los aspectos teóricos que fueron desarrollados en los planteamientos previos, son los encargados de lograr la respectiva interpretación crítica - reflexiva del hecho investigativo, en función de aportar nuevos criterios que sirvan de referencia documental, a otros procesos investigativos, entre ellos se precisan los siguientes:

El cáncer de la mama en las mujeres, es una enfermedad que se origina cuando las células en el seno comienzan a crecer en forma descontrolada. Estas células normalmente forman un tumor que a menudo se puede observar en una radiografía o se puede palpar como una protuberancia (masa o bulto). El tumor es maligno (cáncer) si las células pueden crecer penetrando (invadiendo) los tejidos circundantes o propagándose (metástasis) a áreas distantes del cuerpo. El cáncer de seno ocurre casi exclusivamente en las mujeres, pero los hombres también lo pueden padecer.

La mayoría de las mujeres que presentan cáncer de mama, no tienen signos ni síntomas durante el diagnóstico. Entre los signos y síntomas que precisan el respectivo diagnóstico, se encuentran los 


\section{Complicaciones postoperatorias a las pacientes sometidas a una mastectomía}

Vol. 3, núm. 4., (2019)

Gabriela Cecilia Arroyo Calvopiña; Anthony James Chávez Salazar; Jessica Fernanda Toledo Cascante; Silvia Jimena Zambrano Salvatierra; Fabricio Wladimir Gutiérrez Rivera; Michelle

Madeleyne Salazar Bustamante

siguientes: un bulto que se palpa como un nudo firme o un engrosamiento de la mama o debajo del brazo. Por ello, es importante palpar la misma zona de la otra mama para asegurarse de que el cambio no sea parte del tejido mamario sano de esa área. Cualquier cambio en el tamaño o la forma de la mama. Secreción del pezón que se produce de forma repentina, contiene sangre o se produce solo en una mama. Cambios físicos, como pezón invertido hacia dentro o una llaga en la zona del pezón. Irritación de la piel o cambios en esta, como rugosidades, hoyuelos, escamosidad o pliegues nuevos. Mamas tibias, enrojecidas e hinchadas, con o sin erupción cutánea con rugosidad que se asemeja a la piel de una naranja, llamada piel de naranja. Dolor en la mama; particularmente, que no desaparece. El dolor generalmente no es un síntoma de cáncer de mama, pero debe comunicarse al médico.

En consecuencia, cuando la mujer es diagnosticada con cáncer de mama y dependiendo del estadio en el cual se encuentre el tumor, el cirujano asume la decisión de llevar a cabo una cirugía, identificada como mastectomía, que no es más que extirpar todo el tejido mamario de una mama como una forma de tratar o prevenir el cáncer de mama. Para aquellas mujeres con cáncer de mama en estadio temprano, la mastectomía puede ser una opción de tratamiento. La cirugía de conservación de la mama (tumorectomía), en la que se extirpa solamente el tumor, puede ser otra opción.

Decidir entre practicar una mastectomía y una tumorectomía puede resultar difícil. Ambos procedimientos son igual de eficaces para prevenir la reaparición del cáncer de mama. Sin embargo, la tumorectomía no es una opción para todas las personas que tienen cáncer de mama, y otras prefieren someterse a una mastectomía. Las técnicas más nuevas de mastectomía pueden preservar la piel de la mama y permiten que esta quede con una apariencia más natural después del procedimiento. Este procedimiento también se conoce como «mastectomía con conservación de pie

Cabe agregar que entre las complicaciones que presentan las mujeres son; la infección y dehiscencia de herida quirúrgica, seroma, hematoma y linfedema, todas estas, en su mayoría pueden ser prevenibles, por lo tanto, identificarlas es de suma importancia para las instituciones 


\section{Complicaciones postoperatorias a las pacientes sometidas a una mastectomía}

Vol. 3, núm. 4., (2019)

Gabriela Cecilia Arroyo Calvopiña; Anthony James Chávez Salazar; Jessica Fernanda Toledo Cascante; Silvia Jimena Zambrano Salvatierra; Fabricio Wladimir Gutiérrez Rivera; Michelle Madeleyne Salazar Bustamante

de salud, que coadyuven a la reestructuración de los procesos que están inmersos en la continuidad de la atención. Algunas se presentan a corto plazo, es decir, antes de 40 días y otras después de cuatro a cinco meses clasificadas como tardías.

\section{Bibliografía.}

Angulo, M. (2017). Técnicas y Procedimientos. Caracas: Panapo.

Casillas, N. (2016). ¿Qué es el Cáncer de Mama? Salud, 17-26.

Chavira, A. y. (2019). Complicaciones Postoperatoruias en Mujer con Cirugía por Cáncer de Mama. Scielo, 26-37.

Jiang, J. (2016). Complicaciones después de Mastectomía y Reconstrucción Mamaria. IntraMed, 64-76.

Mata, J. (2018). Método y Ciencia. Caracas: Oasis.

Suárez de Paredes, N. (2010). La Investigación Documental. Paso a Paso . Mérida: Universidad de los Andes.

Torrealba, E. y. (2016). Cáncer de Mama. Factores de Riesgo. Médica, 68-79.

$$
\text { (9) }(1)(0)
$$

\section{RECONOCIMIENTO-NOCOMERCIAL-COMPARTIRIGUAL}

CC BY-NC-SA

ESTA LICENCIA PERMITE A OTROS ENTREMEZCLAR, AJUSTAR Y CONSTRUIR A PARTIR DE SU OBRA CON FINES NO COMERCIALES, SIEMPRE Y CUANDO LE RECONOZCAN LA AUTORÍA Y SUS NUEVAS CREACIONES ESTÉN BAJO UNA LICENCIA CON LOS MISMOS TÉRMINOS. 